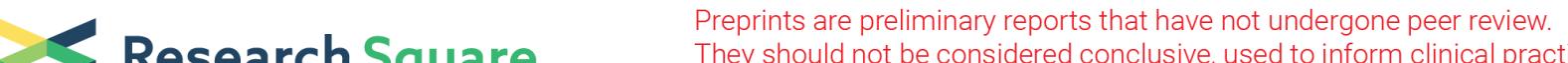 Research Square They should not be considered conclusive, used to inform clinical practice, or referenced by the media as validated information.
}

\section{MicroRNA-2392 Functions as a Tumor Suppressor Gene and Inhibits Malignant Progression of Hepatocellular Carcinoma via Directly Targeting JAG2}

Chunying Liu ( $\nabla$ cyliu@sibcb.ac.cn )

Eastern Hepatobiliary Surgery Hospital https://orcid.org/0000-0002-0113-556X

\section{Bin Sun}

Eastern Hepatobiliary Surgery Hospital

Weidan Ji

Eastern Hepatobiliary Surgery Hospital

Xuejing Lin

Eastern Hepatobiliary Surgery Hospital

Lei Chen

Eastern Hepatobiliary Surgery Hospital

Haihua Qian

Eastern Hepatobiliary Surgery Hospital

Changqing Su

Eastern Hepatobiliary Surgery Hospital

\section{Research Article}

Keywords: microRNA, tumor suppressor, hepatocellular carcinoma, JAG2, proliferation, metastasis

Posted Date: August 4th, 2021

DOI: https://doi.org/10.21203/rs.3.rs-484869/v2

License: (9) (i) This work is licensed under a Creative Commons Attribution 4.0 International License. Read Full License 


\section{Abstract \\ Background}

Dysregulation of microRNA (miRNA) expression in various cancers and their vital roles in malignant progression of cancers are well investigated. Our previous studies have analyzed miRNAs that promote malignant progression of hepatocellular carcinoma (HCC), this study aim to systematically elucidate metastasis suppressor miRNAs in HCC.

\section{Methods}

High-throughput RNA sequencing analysis was used to identify anti-metastatic miRNAs of HCC. The relative expression levels of miRNAs were confirmed by qRT-PCR. The biological functions of miRNAs were studied by CCK8, wound-healing, transwell, colony formation in HCC cells. Circulating tumor cells were enriched from blood samples of HCC patients and cultured by three-dimensional (3D) system. The potential target mRNAs of miRNAs were analyzed by bioinformatics analysis and confirmed by luciferase reporter assay. Liver metastasis model via tail vein injection was further examined in nude mice. KaplanMeier and Cox regression were used to analyze the value of potential target mRNAs on overall survival.

\section{Results}

miR-2392 was significantly down-regulated in HCC. Overexpression of miR-2392 suppressed proliferation, mobility, spheroid formation and maintenance of cancer stem cells (CSC)-like characteristics in HCC cell lines, whereas down-regulation of miR-2392 led to the opposite results. CTCs from HCC patients with lower serum miR-2392 level had stronger cell spheroid formation ability. A negative correlation between the content of miR-2392 in serum and the number of CTC spheroids had been found. We identified Jagged2 (JAG2) as a direct target of miR-2392, miR-2392 inhibited the expression and nuclear accumulation of JAG2 by targeting 3'-UTR of JAG2. HCC cells were treated with LV-miR-2392 inhibitor and JAG2-siRNA to explore the mechanism of miR-2392 and JAG2 on HCC. Down-regulation of JAG2 inhibited the overexpression effects of miR-2392 in vitro and in vivo. JAG2 is highly expressed in HCC and is closely related to poor prognosis and survival of patients.

\section{Conclusions}

Our findings indicated a significant role of the miR-2392/JAG2 axis in suppressing HCC cell growth and aggressiveness, miR-2392 may play a role as a tumor suppressor gene to guide the individualized precise treatment of HCC.

\section{Background}


Cancer incidence and mortality are rapidly growing worldwide. There were 18.1 million new cases and 9.6 million cancer deaths worldwide in 2018 [1]. The latest results showed that 1,898,160 new cancer cases and 608,570 cancer deaths are projected to occur in the United States in 2021 [2]. Primary liver cancer is confirmed by statistics to be the sixth most commonly diagnosed cancer and the fourth leading cause of cancer death, with about 841,000 new cases and 782,000 deaths annually [1]. Hepatocellular carcinoma (HCC) is the most common histological subtype of primary liver cancer (accounting for 75$85 \%$ ) with high invasive, metastatic potential, and has a low survival rate [3]. Due to the insidious onset of HCC patients at early stages and lack of specific biomarkers for early diagnosis, majority of patients had reached advanced stages at their first diagnosis and missed the opportunity of radical resection and liver transplantation. The therapeutic outcomes and quality of life in HCC patients are far from satisfaction. For this reason, it is urgently needed to develop novel effective biomarkers for early diagnosis of HCC.

Circulating tumor cells (CTCS) are tumor cells that shed off the primary tumor, migrate to the blood vessels or lymphatic vessels around the tissues, and exist in the circulatory system [4]. At present, accumulating clinical and basic studies have shown that CTCs might directly participate in metastasis and recurrence of multiple types of malignant tumor, including prostate cancer [5], colorectal cancer [6] and metastatic breast cancer [7]. The metastasis of tumor cells in vivo and in vitro requires the coordinated regulation of tumor microenvironment. The special genetic properties of different tumor cells endow CTCs with the characteristics of survival, proliferation and invasion, and the special tumor microenvironment provides the structural and material basis for metastasis and growth of CTCs. However, the exact regulatory mechanism of survival, migration and colonization of CTCs has not been clarified.

microRNAs (miRNAs) are a class of highly conserved, stable and abundant non-coding RNAs that could directly regulate gene expression at transcriptional or post-transcriptional levels. Finally, they affect the specific protein to perform specific physiological functions in vivo [8]. In recent years, studies on the relationship between miRNAs and tumors have been booming. A variety of miRNAs are identified to act as tumor suppressor or oncogenes, participate in the abnormal changes of biological behavior of tumor cells, and play a crucial role in tumorigenesis and malignant progression $[9,10]$. It is widely recognized that miRNAs mainly derived from the secretion process of animal and plant cells [11]. miRNAs could bind with lipid or lipoprotein components in cells, and secrete into the peripheral blood circulation system in the form of microcapsules. It can also enter the receptor cells through endocytosis, thus playing its specific biological function by regulating target genes. A large number of tumor associated miRNAs have been reported to be tissue-specific and organ specific, and participate in the formation of specific tumor microenvironment. In our previous studies, we found that miRNAs are involved in the formation of tumor microenvironment, and affect the development of HCC by interacting with HCC cells [12, 13]. miRNA could tolerate degradation of RNase, and highly stable in the peripheral circulation system for a long time. As long as we use the conventional total RNA extraction method, we could obtain the concentration of miRNA meeting the experimental requirements, and its expression pattern is tissue-specific. Therefore, the circulating miRNA in peripheral blood has become a target candidate for clinical diagnosis and treatment of HCC [14-16]. 
We speculated according to our previous studies that, on one hand, HCC cells in the primary tumor site and its surrounding tissues could participate in the transformation of HCC microenvironment and malignant biological phenotype of HCC cells by releasing a large number of cancer-related miRNAs, which provides favorable conditions for HCC cells to escape immune surveillance, clonal growth, and spread into blood to form CTCs; on the other hand, circulating miRNAs released into the circulation could also regulate apoptosis-resistance, survival, proliferation and formation of intrahepatic and distant metastasis of CTCs.

\section{Materials And Methods}

\section{Ethics statement}

The use of human tissue samples and clinical data was approved by Committee on Ethics of Medicine, Navy Military Medical University (Shanghai, China). All patients provided the informed written consent. The animal welfare guidelines for the care and use of laboratory animals were followed and the experimental protocol was approved by the Animal Care Committee of Navy Military Medical University (Shanghai, China). The mice were maintained in SPF environment of Experimental Animal Center and sacrificed by anesthetization.

\section{Clinical tissue and cell culture}

HCC tissues were obtained from patients who are treated with surgical resection in Eastern Hepatobiliary Surgery Hospital, and each patient had specific clinical-pathological information. The clinicopathological characteristics of all patients are summarized in Table 1. All patients had postoperative follow-up by the same team of surgeons. A follow-up was conducted as previously described. The median follow-up was 37.1 months (range, 2-60 months). Human normal liver cell lines WRL-68 and L02, HCC cell lines Hep3B, Huh7, HepG2, MHCC97L, PLC/PRF/5 and HCCLM3 (LM3) were cultured in DMEM (Gibco) with10\% FBS, propagated at 37 in $5 \% \mathrm{CO}_{2}$. All the cells used in this study had been identified and confirmed by STR profiling. 
Table 1

Clinical characteristics of the HCC patients according to the expression of JAG2

\begin{tabular}{|c|c|c|c|}
\hline \multirow[t]{3}{*}{ Variables } & \multicolumn{2}{|c|}{ JAG2 expression } & \multirow[t]{3}{*}{$P$ value } \\
\hline & Low & High & \\
\hline & $(n=49)$ & $(n=131)$ & \\
\hline \multicolumn{4}{|l|}{ Sex } \\
\hline Female & 10 & 32 & \multirow[t]{2}{*}{0.57} \\
\hline Male & 39 & 99 & \\
\hline \multicolumn{4}{|c|}{ Age (years) } \\
\hline$\geq 50$ & 18 & 45 & \multirow[t]{3}{*}{0.765} \\
\hline$<50$ & 31 & 86 & \\
\hline \multicolumn{3}{|c|}{ HBV status } & \\
\hline Yes & 28 & 82 & \multirow[t]{2}{*}{0.504} \\
\hline No & 21 & 49 & \\
\hline \multicolumn{4}{|l|}{ Cirrhosis } \\
\hline Yes & 15 & 60 & \multirow[t]{2}{*}{0.066} \\
\hline No & 34 & 71 & \\
\hline \multicolumn{4}{|c|}{ AFP (ng/mL) } \\
\hline$>20$ & 32 & 89 & \multirow[t]{2}{*}{0.738} \\
\hline$\leq 20$ & 17 & 42 & \\
\hline \multicolumn{4}{|c|}{ Tumor size (cm) } \\
\hline$>5$ & 20 & 44 & \multirow[t]{3}{*}{0.367} \\
\hline$\leq 5$ & 29 & 87 & \\
\hline \multicolumn{3}{|c|}{ Tumor number } & \\
\hline Multiple & 11 & 22 & \multirow[t]{2}{*}{0.455} \\
\hline Single & 38 & 109 & \\
\hline \multicolumn{4}{|c|}{ Tumor encapsulation } \\
\hline Yes & 27 & 61 & 0.308 \\
\hline
\end{tabular}




\begin{tabular}{|c|c|c|c|}
\hline \multirow[t]{3}{*}{ Variables } & \multicolumn{2}{|c|}{ JAG2 expression } & \multirow[t]{3}{*}{$P$ value } \\
\hline & Low & High & \\
\hline & $(n=49)$ & $(n=131)$ & \\
\hline No & 22 & 70 & \\
\hline \multicolumn{4}{|c|}{ Vascular invasion } \\
\hline Yes & 18 & 80 & \multirow[t]{2}{*}{0.004} \\
\hline No & 31 & 51 & \\
\hline \multicolumn{4}{|c|}{ TNM stage } \\
\hline $\mid-I I$ & 30 & 55 & \multirow[t]{2}{*}{0.021} \\
\hline III-IV & 19 & 76 & \\
\hline \multicolumn{4}{|c|}{ HBV, hepatitis B virus; AFP, a-fetoprotein; TNM, tumor lymph node metastasis } \\
\hline \multicolumn{4}{|c|}{$p<0.05$ was considered statistically significant. } \\
\hline
\end{tabular}

\section{Cell transfection}

The miR-2392 mimic, inhibitor, and their corresponding negative controls (miR-NC) were purchased from Ribobio (Guangzhou, China). Human siRNA-JAG2 (si-h-JAG2-1, si-h-JAG2-2, si-h-JAG2-3) and si-h-JAG2NC were designed and synthesized by RiboBio (Guangzhou, China). The target sequences of siRNA-JAG2 are listed in Table S1. The optimal working concentration of miR-2392 mimic (100 nM) and inhibitor (200 $\mathrm{nM})$ were selected. miR-2392 mimic (100 nM) and inhibitor (200 nM) were transfected into Huh7 and LM3 respectively, and their effects on the proliferation, migration, invasion and neoplasia were measured according to the manufacturer instructions (RiboBio, Guangzhou, China)

\section{RNA extraction and quantitative reverse transcription-polymerase chain reaction (qRT-PCR)}

Total RNA from HCC serum was obtained by miRNeasy Serum/Plasma Kit (lot. No. 217184, QIAGEN, Germany) according to the instruction of manufacturer. miRNeasy Serum/Plasma Spike-In Control (C.elegans miR-39 miRNA mimic, lot. No. 219610, QIAGEN, Germany) was used as control. Total RNA from HCC tissues and cell lines were obtained by miRNeasy Mini Kit (lot. No. 217004, QIAGEN, Germany) according to the instruction of manufacturer. Then, we reversely transcripted RNA into cDNA according to the instruction of miRcute plus miRNA cDNA First-Strand Synthesis Kit (KR211-02, Tiangen, Beijing, China). Next, cDNA was quantified by application of miRcute enhanced miRNA fluorescence quantitative detection kit (FP411-02, Tiangen, Beijing, China) with gene-targeted or miR-specific primers. We applied the delta-delta $\mathrm{Ct}$ method to conduct quantification as well as calculation of the relative expression of each mRNA or miRNA. The relative expression levels of miRNA and mRNA expression were normalized to U6. Primer sequences are listed in Table S2. Each sample was carried out three times. 


\section{Transcriptome analysis}

Total RNA was extracted from three pairs of fresh human HCC tissues (tumor and para-tumorous tissue) by Trizol reagent (Invitrogen) separately. The RNA quality was checked by Bioanalyzer 2200 (Aligent) and kept at $-80^{\circ} \mathrm{C}$. The RNA with RIN (RNA integrity number) $>8.0$ is acceptable for cDNA library construction. RNA-seq and bioinformatic data analysis were performed by Shanghai Novelbio Ltd.

\section{RNA-seq mapping}

RNA-Seq reads were filtered and mapped to Human genome (GRCh38, NCBI) utilizing HISAT2. HTSeq was used to calculate the gene count. miRNA-Seq reads were filtered and mapped to Human miRNA database (miRBase v21.0) and Human genome (GRCh38, NCBI) to achieve the miRNA expression. Differentially expressed genes analysis was applied utilizing DESeq2. The RNA-seq data was deposited in GEO (Accession code: GSE146719). The target gene of miR-2392 was predicted using the TargetScan database (http://www.targetscan.org/vert_71/). The expression of JAG2 and Notch3 in HCC samples of the Cancer Genome Atlas (TCGA) database was searched using the UALCAN database (http://ualcan.path.uab.edu/analysis.html).

\section{Luciferase activity assay}

The wild-type (WT) sequence containing the predicted target sites of miR-2392 in the 3'UTR of JAG2 mRNA. We mutated the target sites from CCAUCCU to AACGUUG. The wild-type (WT) or mutant (MUT) sequences of JAG2 was inserted into the pGL3 Basic vector (Promega, Madison, WI). Huh7 cells were seeded in 24-well plates at a density of 5000 cells per well. After $24 \mathrm{~h}$, the cells were transiently transfected with a mixture of $0.1 \mu \mathrm{g}$ of luciferase reporter plasmid containing 3 '-UTR segment of JAG2, $0.02 \mu \mathrm{g}$ of Renilla luciferase reporter plasmid, and $0.4 \mu \mathrm{g}$ miRNAs by using X-tremegene HP transfection reagent (Roche, Germany) as described by the manufacturer. Luciferase activity was detected $48 \mathrm{~h}$ after transfection by using the Dual Luciferase Reporter Assay System as described by the manufacturer (Promega, Madison, WI, USA). All samples were independently repeated three times.

\section{Establishment of stable miR-2392 knockdown and overexpressing cells}

miR-2392 was stably knocked down (LV-hsa-miR-2392 inhibitor) and overexpressing (LV-hsa-miR-2392 mimics) in HCC cell lines by means of lentiviral approach which was designed and produced by GeneCopoeia (Rockville, MD). LV-hsa-miR-2392 NC was used as non-target control. The information of sequences is listed in Table S3.

\section{Western blotting}

Protein from fresh HCC samples were obtained with RIPA lysis buffer added with protease inhibitors. After quantification with bicinchoninic acid (BCA) assay (Beyotime, Jiangsu, China), we separated each protein through $10 \%$ SDS-PAGE and then moved them onto PVDF membranes (Millipore, USA). Then, samples were blocked with $5 \%$ nonfat milk. After incubation with primary antibodies against GAPDH and 
JAG2 (1:200, ab109627, Abcam, UK) and secondary antibodies, protein levels were detected with Image J (GE Healthcare Life Sciences). Each sample was analyzed three times.

The total cellular protein and the nuclear protein were extracted according to instructions of nuclear and cytoplasmic extraction reagents kit (Beyotime, Jiangsu, China). The protein concentrations were determined by bicinchoninic acid (BCA) assay (Beyotime, Jiangsu, China). $20 \mu \mathrm{g}$ of protein was loaded per lane and separated by $10 \%$ SDS-PAGE and electrotransferred to PVDF membranes (Millipore, USA). GAPDH was used as internal control. Antibody information: PCNA (1:1000, ab92552, Abcam, UK), MMP2 (1:1000, ab92536, Abcam, UK), MMP9 (1:1000, ab76003, Abcam, UK), Vimentin (1:1000, ab8979, Abcam, UK).

\section{Cell proliferation assay}

Cell proliferation assay was conducted with Counting Kit-8 (CCK-8) (Beyotime, Jiangsu, China). 5000 cells (transfected with miR-NC, miR-2392 mimic, miR-2392 inhibitor) were planted into 96-well plates for $24 \mathrm{~h}$, $48 \mathrm{~h}$ and $72 \mathrm{~h}$ respectively. Then $10 \% \mathrm{CCK}-8$ solution was added. The absorbance of each sample was assessed by a microplate reader set at $450 \mathrm{nM}$. Each sample was performed for three times.

\section{Cell migration and invasion assay}

The methods of cell migration and invasion assays were constructed as previously described. Confluent cell monolayers which transfected with miR-2392 mimic, inhibitor and miR-NC in 6-well plates were wounded by manual scraping with a sterile pipette tip. The imagines were analyzed by Image $\mathrm{J}(\mathrm{NIH})$. Cell invasion assay was performed on Matrigel-coated transwell chambers (Corning, NY, USA) with the treatment of miR-2392 mimic, inhibitor and miR-NC.

\section{Colony formation assay}

Cells (2000 cells/well) which transfected with miR-NC, miR-2392 mimic and miR-2392 inhibitor were seeded in each well of a six-well cell culture plate. After 2 weeks, the plates were fixed in $4 \%$ paraformaldehyde and stained with $1 \%$ crystal violet. The colony numbers were counted to assess cell proliferation. The assays were performed in three independent experiments.

\section{Immunohistochemistry}

Tissue microarrays consisting of 180 cases of HCC tissues and matched para-cancerous tissues were constructed. Tissue sections were deparaffinized, rehydrated, and treated with $3 \% \mathrm{H}_{2} \mathrm{O}_{2}$ for 15 min to inhibit endogenous peroxidase activity. Following heat-induced epitope retrieval in $10 \mathrm{mM}$ citrate buffer $(\mathrm{pH} 6.0)$ in a microwave for $15 \mathrm{~min}$, the slides were incubated at $4{ }^{\circ} \mathrm{C}$ overnight with a pre-diluted primary antibody (JAG2, 1:200, ab109627; Notch3, 1:200, ab23426, Abcam, UK). After incubation with a secondary antibody, the signal was developed with 3,3'-diaminobenzidine tetrachloride. 
The staining scores were evaluated by two individuals in a blinded fashion. A quick scoring system from 0 to 12 that combined the intensity and percentage of the positive signal was used. Briefly, a signal of 0 indicated no staining, 1 indicated weak staining, 2 indicated intermediate staining and 3 indicated strong staining. Percentage scores were assigned as follows: 0 corresponded to $0 \%, 1$ to $1-25 \%, 2$ to $26-50 \%, 3$ to $51-75 \%$, and 4 to $>75 \%$. The median value of total staining scores was identified as the optimal cut-off value. If the evaluated score was lower than the median, the indicator expression of in those HCC samples was classified as low; otherwise, it was classified as high $[17,18]$.

\section{CTC enrichment}

CTC enrichment of whole blood samples was conducted according to the method previously described. Briefly, following density gradient centrifugation (Ficoll-Paque PLUS,GE Healthcare, WI, USA), CTCs were enriched by extracting CD45-expressing leukocytes with magnetically beads labeled anti-CD45 monoclonal antibody (130-045-801, Miltenyi Biotec, Germany) according to the instructions. The remaining cells were cyto-centrifuged on polylysine-coated slides for identification and enumeration of CTCs, or resuspended in $150 \mu \mathrm{L}$ Dulbecco modified Eagle medium (DMEM) (high glucose) (Thermo Fisher Scientific, MA, USA) for the in vitro three-dimensional (3D) culture of CTCs.

\section{Three-dimensional (3D) culture in vitro}

The enriched CTCs were cultured using a 3D cell culture assay. Briefly, Matrigel (BD Biosciences, San Jose, CA, USA) was thawed according to the manufacturer's instructions and mixed equally with the CTCcontaining DMEM. When HCC cell lines are used for $3 \mathrm{D}$ culture, $1 \times 10^{3}$ cells suspended in DMEM were mixed with Matrigel. The prepared mixture was then incubated in a 24 -well plate for $30 \mathrm{~min}$ at $37^{\circ} \mathrm{C}$. Subsequently, $500 \mu \mathrm{L}$ DMEM supplemented with $10 \%$ fetal bovine serum (FBS) were added to the plate, and the media was supplied every two days during culture. Spheroid formation was observed under a microscope every day and counted on day 7. A spheroid was defined as 3D cell structure $>100 \mu \mathrm{m}$ in diameter that was determined using images taken on a microscope.

\section{Immunofluorescence staining}

Cell spheroids were slightly detached, picked, and fixed on glass slides with $4 \%$ paraformaldehyde. Blocking was performed with normal goat serum. Slides were incubated for $1 \mathrm{~h}$ with the primary antibodies, including the mouse anti-human CPS1 (1:200, ab110303, Abcam, UK), mouse anti-human ASGPR (1:400, sc-52623, Santa Cruz, USA), rabbit anti-human EpCAM (1:200, ab213500, Abcam, UK) and rabbit anti-human CD133 (1:200, \#64326, CST, USA). CPS1 and ASGPR antibodies were used for detection of hepatocytes $(29,30)$, EpCAM and CD133 antibodies were used for detection of stem-like cells. After washing 3 times with PBS, the secondary antibodies, including the Cy3-conjugated goat antimouse/rabbit IgG (Beyotime, China), Alexa Fluor 647-conjugated goat anti-mouse/rabbit IgG (Beyotime, China) or Alexa Fluor 488-conjugated goat anti-rabbit IgG (Beyotime, China) were added and incubated at room temperature for $40 \mathrm{~min}$. Cells were then counterstained with 6-diamidino-2-phenylindole (DAPl; 
Sigma-Aldrich, St. Louis, MO, USA) and the images were captured using an Olympus-IX71 fluorescent microscope (Olympus Inc., Center Valley, PA, USA).

\section{In vivo tumor growth and metastasis model}

BALB/c male nude mice 4-6 weeks old were purchased from Shanghai Laboratory Animal Center, Chinese Academy of Sciences (Shanghai, China). Mice were raised under pathogen-free conditions. All in vivo experiments were done according to approved protocols from the Institutional Animal Care and Use Committees, according to national and institutional guidelines. Briefly, to establish in vivolung metastasis model, $2 \times 10^{6}$ viable cells (LV-hsa-miR-2392 inhibitor or LV-hsa-miR-2392 NC) were injected into the tail veins of mice. One week after inoculation, si-h-JAG2-3 (2'OMe $+5{ }^{\prime} \mathrm{Chol}$ modified) was injected into the tail vein three times a week $(10 \mathrm{nmol} /$ time/mouse) for treatment. After monitoring for 6 weeks, mice were sacrificed, and their lungs were dissected, fixed with phosphate-buffered neutral formalin, and prepared for standard histological examination.

\section{Statistical analysis}

Data was reported as mean \pm SD. Data analysis was conducted by IBM SPSS Statistics Version 19.0 and Graphpad Prism 5. Analysis of Variance (ANOVA) was used for comparison between groups. The gray value was analyzed by Image-Pro Plus 6.0 software. Survival analysis was estimated by the Kaplan-Meier survival method. Variables with a P value of $<0.05$ in the univariate analyses were further tested using multivariate Cox proportional hazards model. Results were determined to be statistically significant when $P<0.05$.

\section{Results}

\section{miRNA and mRNA expression profiling in HCC tissues}

miRNAs could exist for a long time in the peripheral circulatory system which endows them with the potential to be ideal biomarkers for human diseases. In the present study, to explore novel biomarkers of HCC, we first explored the expression profiles of mRNAs and miRNAs in three pairs of HCC patients (tumor tissues and non-tumorous tissues) by high-throughput RNA sequencing. A total of 1,024 mRNAs were found to be significantly dysregulated (636 up-regulated and 388 down-regulated) (Supplementary Fig. $1 \mathrm{~A}$ and $1 \mathrm{~B}), 50$ miRNAs were found to be significantly dysregulated (27 up-regulated and 23 downregulated) in the HCC tissues compared with the non-tumorous tissues (Fig. 1A and 1B). Significant differences in mRNA and miRNAs between HCC tissues and non-tumorous tissues were classified as fold change $\geq 2$ or fold change $\leq 0.5$, FDR $<0.05$, as determined by having to satisfy both of the above conditions. Two of the up-regulated miRNAs (miR-206 and miR-6812-5p) and two of the down-regulated miRNAs (miR-2392 and miR-96-5p) were selected for validation by qRT-PCR.

\section{Validation Of Differentially Expressed Mirnas By Qrt-pcr}


To validate the result, two of the down-regulated miRNAs (miR-2392 and miR-6812-5p) and two of the upregulated miRNAs (miR-206 and miR-96-5p) were selected for validation by qRT-PCR. We analyzed miRNAs expression in paired HCC tissues and corresponding non-tumorous tissues, and found that the expression level of miR-2392 was markedly lower in HCC tissues, which was in consistence with the detection of miR-2392 in serum of HCC patients (Fig. 1C and 1D). The expression of the other three miRNAs in paired HCC and para-cancerous tissues was consistent with the sequencing data, but the serum content was different (Supplementary Fig. 1C and 1D). The results showed that the expression profile of miR-2392 was consistent with those from the sequencing results. Collectively, these data suggest the accuracy and reproducibility of the RNA sequencing findings.

\section{Bioinformatics analysis of the potential role of miR-2392 in HCC}

Gene ontology analysis was performed to facilitate elucidating the biological implications of differentially expressed miRNA. Pathway analysis was used to find out the significant pathway of the differential genes according to KEGG database. Go analysis and pathway analysis results revealed that the changes of Notch signaling pathway is one of the most significant in all signaling pathways (Fig. 1E). In order to further explore the potential functions of miR-2392 in HCC patients, we performed a miRNAmRNA-pathway network analysis from our RNA-seq data were selected (Fig. 1F). We found that there were several potential target mRNAs of miR-2392 including JAG2 (a key regulator of Notch signaling pathway), NGFR, IGF2, IL7R, etc. Protein-protein interaction network (PPI network) results predicted that JAG2 and Notch3 present the highest score (Fig. 1G). These results suggested that JAG2/Notch3 signaling pathway may be involved in the biological regulation of miR-2392 in HCC.

\section{miR-2392 was down-regulated in HCC cell lines which promoted the proliferation, migration, invasion, clonal formation and spheroid formation of HCC cells in vitro}

By qRT-PCR, the expression of miR-2392 was down-regulated in HCC cells compared to normal liver cells (Fig. 1H). We chose Huh7 (relatively low endogenous expression) and LM3 (relatively high endogenous expression) as cell model for subsequent experiments according to the expression profiles of miR-2392 in HCC cells. To investigate the potential role of miR-2392 in HCC cells, we used miR-2392 mimic and inhibitor to transfect two HCC cell lines (Huh7 and LM3). The optimal concentration of miR-2392 mimic and inhibitor has been selected and verified (Supplementary Fig. 2).The results suggested that downregulation of miR-2392 promoted the proliferation, invasion and neoplasia ability of LM3. On the contrary, up-regulation of miR-2392 inhibited the proliferation, invasion and neoplasia ability of Huh7 (Fig. 2A-2D). Previous study indicated that miRNA could maintain CSC-like phenotype in tumor cells. A tumor sphere formation assay was used to explore the effect of miR-2392 on CSC self-renewal properties in HCC. Our results revealed that miR-2392 inhibitor-transfected HCC cells (LM3) formed more and larger spheroids than miR-2392 NC group and blank group. On the contrary, the miR-2392 mimic-transfected HCC cells (Huh7) formed less and smaller spheroids than miR-2392 NC group and blank group (Fig. 3A). The protein expression level of stem cell related genes, EpCAM and CD133 were further verified by cellular immunostaining. The present data indicated that EpCAM could be observed in almost all spheroids, but 
down-regulation of miR-2392 promotes the expression of CD133 in LM3 cells. Transfection of miR-2392 mimic decreased the expression level of CD133 in Huh cells (Fig. 3B). Overall, these data suggested that down-regulation of miR-2392 might be closely related to the malignant phenotype (proliferation and motility) and maintenance of CSC-like characteristics of HCC cells in vitro.

\section{Low level of miR-2392 promotes CSC-like properties of HCC CTCs in vitro}

According to the concentration of miR-2392 in sera of HCC patients, all patients could be divided into two groups: miR-2392 high group (mean concentration $\geq 100,000$ ) and miR-2392 low group (mean concentration < 100,000). Three dimensional culture of CTCs isolated from HCC patients in vitro for 14 days. We analyzed the correlation between the number of CTC spheroids and the content of miR-2392 in sera of HCC patients. The results found that the CTCs from HCC patients with lower serum miR-2392 level had stronger cell spheroid formation ability. In addition, a negative correlation between the content of miR-2392 in serum and the number of CTC spheroids was observed $\left(P=0.0002, R^{2}=-0.2539\right)$ (Fig. $3 C)$. The results of cellular immunofluorescence also confirmed that low level of miR-2392 promotes the CSC-like properties of HCC CTCs in vitro (Fig. 3D).

\section{Jag2 Is A Direct Target Of Mir-2392}

Previous RNA-seq data and bioinformatics analysis suggested that JAG2 may be the downstream target of miR-2392. We predicted miR-2392 potential regulatory target genes by Targetscan (http://www.targetscan.org/vert_71/). The result revealed that miR-2392 has a direct binding site with JAG2 (Fig. 4A). To verify JAG2 was a downstream target of miR-2392, we carried out a luciferase reporter assay to corroborate the direct interaction between JAG2 and miR-2392. WT or MUT 3'-UTR target sequences were cloned into a luciferase reporter vector (Fig. 4B). Our results showed that miR-2392 inhibited the luciferase activity of the WT 3'-UTR of JAG2 (Fig. 4C). These results confirmed that JAG2 was a direct target of miR-2392.

\section{miR-2392 regulates the transcription of downstream genes by inhibiting the nuclear translocation of JAG2 protein}

Cellular immunofluorescence assay was performed to measure the expression and localization of JAG2 protein in HCC cells treated with miR-2392. The results indicated that miR-2392 mimic induced a decrease in the expression of JAG2, which was accompanied by a less nuclear accumulation of JAG2. By contrast, pretreatment with miR-2392 inhibitor led to an increase in the expression of JAG2, which was accompanied by a greater amount of nuclear accumulation of JAG2 (Fig. 4D). We extracted proteins from the cytoplasm and nucleus of HCC cells which pretreated with miR-2392 mimic and inhibitor, respectively. The results of western blotting confirmed that miR-2392 mimic decreased the expression and nuclear translocation of JAG2. Conversely, miR-2392 inhibitor significantly increased the expression and nuclear translocation of JAG2 in HCC cells (Fig. 4E). 
Notch signaling pathway target genes vary in different tissue and different cells, such as HES and HEY families, MMP2, MMP9, cyclin D1, Her2, c-Myc, p21 and cell apoptosis-associated genes. Notch precisely regulates cell differentiation, proliferation and tumorigenesis might by modulating these genes expression. Our results showed that down-regualtion of miR-2392 increased MMP2, MMP9, PCNA and Vimentin expression. To the opposite, up-regualtion of miR-2392 decreased MMP2, MMP9, PCNA and Vimentin expression. Therefore, we speculate that low level of miR-2392 enhanced the proliferation ability of HCC cells and accelerated the degradation of extracellular matrix (ECM), which will facilitate the invasion and metastasis of HCC cells. Collectively, these data provide strong biochemical evidence that low level of miR-2392 significantly increased the expression and nuclear translocation of JAG2 which activates Notch signaling pathway to facilitate HCC growth and metastasis.

\section{Relationship between the mRNA levels of JAG2/Notch3 and the clinicopathological parameters of patients with HCC}

Using the UALCAN (Data from TCGA database) (http://ualcan.path.uab.edu), we compared the mRNA expression of JAG2 and Notch3 between HCC tissues and normal liver tissues $(n=371)$. The results indicated that the expression levels of JAG2 and Notch3 were higher in HCC tissues than in normal tissues (Fig. 5A; Supplementary Fig. 3A).

Next, we performed immunohistochemistry (IHC) to test JAG2 and Notch3 protein expression in HCC tissues and their counterparts. We found that JAG2 and Notch3 proteins were more highly expressed in $\mathrm{HCC}$ tissues than in the normal tissues (Fig. 5B and Supplementary Fig. 3B). Furthermore, western blot assay was carried out to detect the expression of JAG2 and Notch3 in extracted protein from fresh HCC tissues ( $n=32$ ) (Fig. 5C, 5D; Supplementary Fig. 3C). The results suggested that the expression levels of JAG2 and Notch3 was higher in HCC tissues than in non-tumorous tissues.

According to the expression pattern and evaluation of JAG2 in HCC tissues, the average score is 6 (Fig. 5B). $131 \mathrm{HCC}$ samples which score was higher than the average score was classified as high expression group. Otherwise, $49 \mathrm{HCC}$ samples which score was lower than the average score was classified as low expression group. As summarized in Table 1, the correlation between JAG2 protein expression and various clinicopathological characteristics was investigated, as well as follow-up data, were collected and recorded. The median follow-up period was 37.1 months (range, 2-60 months). Subsequent association analysis determined that high expression levels of JAG2 in HCC tissues significantly correlated with vascular invasion $(P=0.008)$ and TNM stage $(P=0.034)$. The results of the present study indicate that abnormal expression levels of JAG2 may be associated with the recurrence and metastasis of HCC. As shown in Fig. 5E, HCC with high expression of JAG2 was associated with a significantly lower overall survival rate $(P=0.0359)$ and recurrence-free survival $(P=0.0262)$ than was HCC with low expression of JAG2.

Based on the univariate analysis, vascular invasion, TNM stage and JAG2 expression were selected to be further tested for multivariate analysis (Tables 2 and 3). The results indicated that JAG2 was one of the 
independent favorable factors for overall survival and recurrence, as were advanced TNM stage, and vascular invasion. 
Table 2

Univriate and multivariate analyses of the factors associated with overall survival in 180 HCC patients.

\begin{tabular}{|c|c|c|c|c|}
\hline \multirow[t]{3}{*}{ Features } & \multirow[t]{3}{*}{ Univariate $P$} & \multicolumn{3}{|c|}{ Overall survival } \\
\hline & & \multirow[t]{2}{*}{ HR } & Multivariate & \multirow[t]{2}{*}{$\mathbf{P}$} \\
\hline & & & $95 \% \mathrm{Cl}$ & \\
\hline Sex & 0.959 & & & NA \\
\hline \multicolumn{5}{|l|}{ Male versus female } \\
\hline Age & 0.783 & & & NA \\
\hline \multicolumn{5}{|l|}{$\geq 50$ versus $<50$} \\
\hline HBV status & 0.204 & & & NA \\
\hline \multicolumn{5}{|l|}{ yes versus no } \\
\hline Cirrhosis & 0.374 & & & NA \\
\hline \multicolumn{5}{|l|}{ yes versus no } \\
\hline AFP (ng/mL) & 0.430 & & & NA \\
\hline \multicolumn{5}{|l|}{$>20$ versus $\leq 20$} \\
\hline Tumor size (cm) & 0.229 & & & NA \\
\hline \multicolumn{5}{|l|}{$>5$ versus $\leq 5$} \\
\hline Tumor number & 0.139 & & & NA \\
\hline \multicolumn{5}{|l|}{ Multiple versus single } \\
\hline Tumor encapsulation & 0.134 & & & NA \\
\hline \multicolumn{5}{|l|}{ yes versus no } \\
\hline Vascular invasion & $<0.001$ & 3.645 & $(0.877-15.144)$ & 0.075 \\
\hline \multicolumn{5}{|l|}{ yes versus no } \\
\hline TNM stage & $<0.001$ & 7.433 & $(1.830-30.188)$ & 0.005 \\
\hline \multicolumn{5}{|l|}{ I-II versus III-IV } \\
\hline JAG2 expression & 0.033 & 1.472 & $(0.638-3.395)$ & 0.365 \\
\hline low versus high & & & & \\
\hline
\end{tabular}

Univeriate analysis: Kaplan-Meier method; multivariate analysis: Cox proportional hazards regression model.

Abbreviations: HBV: hepatitis B virus; HR: hazard ratio; Cl: confidence interval; NA: not adopted 
Page 16/30 
Table 3

Univriate and multivariate analyses of the factors associated with recurrence in $180 \mathrm{HCC}$ patients.

\begin{tabular}{|c|c|c|c|c|}
\hline \multirow[t]{2}{*}{ Features } & \multirow[t]{2}{*}{ Univariate $P$} & \multicolumn{3}{|c|}{ Recurrence } \\
\hline & & \multirow[t]{2}{*}{ HR } & Multivariate & \multirow[t]{2}{*}{$\mathbf{P}$} \\
\hline & & & $95 \% \mathrm{Cl}$ & \\
\hline Sex & 0.625 & & & NA \\
\hline \multicolumn{5}{|l|}{ Male versus female } \\
\hline Age & 0.789 & & & NA \\
\hline \multicolumn{5}{|l|}{$\geq 50$ versus $<50$} \\
\hline HBV status & 0.116 & & & NA \\
\hline \multicolumn{5}{|l|}{ yes versus no } \\
\hline Cirrhosis & 0.296 & & & NA \\
\hline \multicolumn{5}{|l|}{ yes versus no } \\
\hline AFP (ng/mL) & 0.493 & & & NA \\
\hline \multicolumn{5}{|l|}{$>20$ versus $\leq 20$} \\
\hline Tumor size (cm) & 0.852 & & & NA \\
\hline \multicolumn{5}{|l|}{$>5$ versus $\leq 5$} \\
\hline Tumor number & 0.170 & & & NA \\
\hline \multicolumn{5}{|l|}{ Multiple versus single } \\
\hline Tumor encapsulation & 0.213 & & & NA \\
\hline \multicolumn{5}{|l|}{ yes versus no } \\
\hline Vascular invasion & $<0.001$ & 3.567 & $(0.624-20.395)$ & 0.153 \\
\hline \multicolumn{5}{|l|}{ yes versus no } \\
\hline TNM stage & $<0.001$ & 7.198 & $(1.284-40.341)$ & 0.025 \\
\hline \multicolumn{5}{|l|}{ I-II versus III-IV } \\
\hline JAG2 expression & 0.022 & 1.703 & $(0.724-4.007)$ & 0.222 \\
\hline \multicolumn{5}{|l|}{ low versus High } \\
\hline $\begin{array}{l}\text { Univeriate analysis: Ka } \\
\text { model. }\end{array}$ & method; multiv & nalysis & x proportional ha & yression \\
\hline
\end{tabular}


miR-2392 suppresses tumor metastasis of HCC cells in vivo and in vitro

To further validate inhibiting roles of miR-2392 in HCC progression, we established an experimental in vivo metastasis assay by tail injection of stably knockeddown-miR-2392 LM3 cells (LV-hsa-miR-2392 inhibitor) which had been validated by cellular immunostaining (Supplementary Fig. 4). Effective JAG2siRNA had been screened in vitro before (Supplementary Fig. 5). The experimental groups were as follows: (1)LV-miR2392-NC; (2)LV-miR2392-inhibitor;(3)LV-miR2392-inhibitor + JAG2-siRNA-NC;(4)LVmiR2392-inhibitor + JAG2-siRNA. As demonstrated in Fig. 6, the numbers of micrometastatic nodules in the lungs of mice were markedly increased in LV-miR2392-inhibitor group (17.2 \pm 12.07$)$ and LV-miR2392inhibitor + JAG2-siRNA-NC group $(20.4 \pm 10.26)$ as compared to that in the control groups $(4.6 \pm 2.88)$. Treatment of JAG2-siRNA could restore the inhibitory effect of miR-2392 on HCC cell metastasis $(5.2 \pm$ 1.64). Meanwhile, the results of cell migration and invasion assay by stably knocking down/overexpressing-miR-2392 HCC cells confirmed that miR-2392 could significantly inhibit the metastasis of HCC cells. Supplementing JAG2 (100nM) in miR-2392 inhibition group could restore inhibitory effect of miR-2392 on HCC cell metastasis (Supplementary Fig. 6). These results suggested that miR-2392 plays a crucial role in inhibiting tumor metastasis of HCC in vivo and in vitro.

\section{Discussion}

HCC metastasis and recurrence is the main cause of HCC-related death. However, its mechanism remains poorly understood. Further study on the key factors of tumor microenvironment (TME) may be helpful. In an attempt to identify TME-related factors (such as miRNAs) that are specific for HCC progression, we recently conducted a high-throughput RNA sequencing by comparing primary tumors with their matched non-tumorous tissues.

In this study, we found a group of cancer-related miRNAs (miR-2392, miR-6812, miR-96-5p, miR-206) by high-throughput RNA sequencing of freshly collected HCC tissues and paired non-tumorous tissues. The results of subsequent validation in serum and tissue samples of HCC patients showed that the expression level of miR-2392 was significantly lower than that of non-tumorous tissues which was most consistent with the high-throughput RNA sequencing results. We found that miR-2392 could significantly inhibit the ability of proliferation, migration, invasion and spheroidization of HCC cells in vitro. miR-2392 might act as a tumor suppressor in HCC.

Circulating tumor cells (CTCs) disseminated in circulation system are liable to suffer apoptosis by blood shear stress and anoikis. Therefore, the surviving CTCs maybe the key factor of recurrence and metastasis. Likely, the tumor microenvironment could provide clues for elucidating the survival mechanism of CTCs. The amplification and culture of CTCs in vitro has been a technical bottleneck for a long time due to the extremely rare number of CTCs. We successfully expand CTCs by using the threedimensional cell culture system through continuous attempts. Correlation analysis of the number of CTC spheroids and the content of miR-2392 in serum of HCC patients. Our present results indicated that the 
CTCs from HCC patients with lower serum miR-2392 level had stronger cell spheroid formation ability. Furthermore, a negative correlation between the content of miR-2392 in serum and the number of CTC spheroids was observed $\left(P=0.0002, R^{2}=-0.2539\right)$. These results suggested that miR-2392 might be one of the crucial factors affecting the survival of CTCs in vitro.

Next, we focused on the exploration for miR-2392 targeted genes and related signaling pathways. Bioinformatics analysis of the potential role of miR-2392 in HCC based on the high-throughput RNA sequencing data. Go analysis and pathway analysis results revealed that the changes of Notch signaling pathway is one of the most significant in all signaling pathways. miRNA-mRNA-pathway network analysis and protein-protein interaction network (PPI network) results predicted that JAG2 and Notch3 participate in miR-2392 signaling regulatory network.

The Notch pathway directly couples events at the cell membrane with the regulation of transcription, which includes 4 Notch receptors (Notch1-4) and 5 Notch ligands (Delta-like ligands (DII1, DII3, and DII4) and 2 serrate-like ligands (Jagged 1 and Jagged 2)) [19]. Activation of Jagged2 (JAG2) is frequently observed in several tumors and involved in the malignant biological behavior of tumor cells [20, 21]. However, the mechanism that causes JAG2 activation in HCC remains to be unraveled. First of all, we compared the mRNA expression of JAG2 and Notch3 between HCC tissues and normal liver tissues ( $\mathrm{n}=$ 371) by using the UALCAN (Data from TCGA database) (http://ualcan.path.uab.edu). The results indicated that the expression levels of JAG2 and Notch3 were higher in HCC tissues than in normal tissues. IHC and western blot assay were used to measure the expression pattern of JAG2 in HCC tissues. Our present results revealed that JAG2 was higher in HCC tissues than in non-tumorous tissues which was one of the independent favorable factors for overall survival and recurrence, as were advanced TNM stage, and vascular invasion. Furthermore, we predicted miR-2392 potential regulatory target genes by Targetscan (http://www.targetscan.org/vert_71/). The result revealed that miR-2392 has a direct binding site with JAG2. We carried out a luciferase reporter assay to verify the direct interaction between JAG2 and miR-2392. The results confirmed that miR-2392 inhibited the luciferase activity by combining 3'-UTR of JAG2. The results of cellular immunofluorescence and western blot assay also confirmed that downregulation of miR-2392 dramatically increased the expression and nuclear translocation of JAG2. Moreover, the results of pulmonary metastasis model suggested that miR-2392 significantly inhibited metastasis of HCC cells. Deprive of the expression of miR-2392 remarkably enhanced the colonization and growth of HCC cells in the lung. Inhibition of JAG2 expression by siRNA could restore the inhibitory function of miR-2392. The identification of a model of miR-2392/JAG2 interaction may promote the understanding of the underlying mechanism of HCC metastasis. Collectively, the findings from the present study provide an overview, as depicted in Fig. 7. Downstream target genes of Notch signaling pathway vary in different tissue and cells, such as HES and HEY families, MMP2, MMP9, cyclin D1, Her2, c-Myc, p21 and cell apoptosis-associated genes which participate in regulating cell differentiation, proliferation and tumorigenesis [22, 23]. We further found that inhibition of miR-2392 increased the expression of MMP2, MMP9, PCNA and Vimentin which could enhance the proliferation ability of HCC cells, accelerate the degradation of extracellular matrix (ECM), eventually facilitate the invasion and 
metastasis of HCC cells. Additionally, the potential mechanism of MMP2, MMP9, PCNA and Vimentin regulated by miR-2392 will be further analyzed. According to our present results, we speculate that the decrease of miR-2392 expression and concentration in HCC leads to the increase of JAG2 protein expression and nuclear accumulation, which leads to transcriptional changes of a series metastasis related genes, and ultimately promotes HCC cell metastasis.

\section{Conclusions}

Accordingly, we identified miR-2392 functions as a tumor suppressor gene which is down-regulated in $\mathrm{HCC}$, and correlates with proliferation, metastasis, spheroid formation and maintenance of CSC-like characteristics of HCC cells via directly targeting JAG2. These findings provide a new mechanism for understanding $\mathrm{HCC}$ metastasis and miR-2392 may be a potential candidate in the individualized precise treatment of HCC.

\section{Abbreviations}

miRNA, microRNA; JAG2, Jagged2; CTCs, Circulating tumor cells; HCC, hepatocellular carcinoma; TCGA, the Cancer Genome Atlas database; Go analysis, gene ontology analysis; PPI, protein \& protein interaction; 3D culture, three-dimensional culture; CSC, cancer stem cell; IF, immunofluorescence; IHC, immunohistochemistry; qRT-PCR, quantitative real time-PCR.

\section{Declarations}

\section{Acknowledgements}

We would like to show sincere appreciation to the reviewers for critical comments on this article.

\section{Funding}

This work was supported by grants from National Key R\&D Program of China (2018YFA0900902), National Natural Science Foundation of China $(81802736,81672373)$.

\section{Availability of data and materials}

The datasets generated and/or analysed during the current study are available.

\section{Consent for publication}

Not applicable.

\section{Ethics approval and consent to participate}

The use of human tissue samples and clinical data was approved by Committee on Ethics of Medicine, Navy Military Medical University (Shanghai, China). The animal welfare guidelines for the care and use of 
laboratory animals were followed and the experimental protocol was approved by the Animal Care Committee of Navy Military Medical University (Shanghai, China).

\section{Competing interests}

The authors declare no potential conflicts of interest.

\section{Authors' contributions}

Bin Sun: Methodology, data curation, original draft preparation. Weidan Ji: Methodology, visualization, data curation. Chunying Liu \& Xuejing Lin: Data curation, software, validation. Lei Chen: Methodology, investigation. Haihua Qian: Methodology, validation, software. Changqing Su: Conceptualization, supervision and reviewing and editing.

\section{References}

1 Bray F, Ferlay J, Soerjomataram I, Siegel RL, Torre LA, Jemal A. Global cancer statistics 2018: GLOBOCAN estimates of incidence and mortality worldwide for 36 cancers in 185 countries. CA Cancer J Clin. 2018; 68, 394-424.

2 Siegel RL, Miller KD, Fuchs HE, Jemal A. Cancer Statistics, 2021. CA Cancer J Clin. 2021; 71, 7-33.

3 Qiu L, Xu H, Ji M, Shang D, Lu Z, Wu Y, Tu Z, Liu H. Circular RNAs in hepatocellular carcinoma: Biomarkers, functions and mechanisms. Life Sci. 2019; 231, 116660.

4 Massagué J, Obenauf AC. Metastatic colonization by circulating tumour cells. Nature.2016; 529, 298306.

5 Palermo G, Bassi P, Racioppi M, Recupero SM, Sacco E, Campetella M, Canu G, Pinto F. Circulating tumor cells as prognostic biological marker in different stages prostate cancer and the effect of different therapeutic approaches on their expression. Minerva Urol Nefrol. 2020; 72, 214-222.

6 Malara N, Trunzo V, Foresta U, Amodio N, De Vitis S, Roveda L, Fava M, Coluccio M, Macrì R, Di Vito A, et al. Ex-vivo characterization of circulating colon cancer cells distinguished in stem and differentiated subset provides useful biomarker for personalized metastatic risk assessment. J Transl Med. 2016; 14, 133.

7 Cristofanilli M, Budd GT, Ellis MJ, Stopeck A, Matera J, Miller MC, Reuben JM, Doyle GV, Allard WJ, Terstappen LW, Hayes DF. Circulating tumor cells, disease progression, and survival in metastatic breast cancer. N Engl J Med. 2004; 351, 781-791.

8 Maltby S, Plank M, Ptaschinski C, Mattes J, Foster PS. MicroRNA function in mast cell biology: proticols to characterize and modulate microRNA expression. Methods Mol Biol. 2015; 1220, 287-304. 
9 Hata A, Lieberman J. Dysregulation of microRNA biogenesis and gene silencing in cancer. Sci Signal. $2015 ; 8$, re3.

10 Cheng CJ, Bahal R, Babar IA, Pincus Z, Barrera F, Liu C, Svoronos A, Braddock DT, Glazer PM, Engelman DM, et al. MicroRNA silencing for cancer therapy targeted to the tumor microenvironment. Nature. 2015; 518, 107-110.

11 Ohshima K, Inoue K, Fujiwara A, Hatakeyama K, Kanto K, Watanabe Y, Muramatsu K, Fukuda Y, Ogura $\mathrm{S}$, Yamaguchi $\mathrm{K}$, et al. Let-7 microRNA family is selectively secreted the extracellular environment via exosomes in a metastatic gastric cancer cell line. PLoS One. 2010; 5, e13247.

12 Li X, Su Y, Sun B, Ji W, Peng Z, Xu Y, Wu M, Su C. An artificial interfering IncRNA mediated by oncolytic adenovirus competitively consumes OncomiRs to exert antitumor efficacy in hepatocellular carcinoma. Molecular Cancer Therapeutics. 2016; 15,1436-1451.

13 Bao L, Yan Y, Xu C, Ji W, Shen S, Xu G, Zeng Y, Sun B, Qian H, Chen L, et al. MicroRNA-21 suppresses PTEN and hsulf-1 expression and promotes hepatocellular carcinoma progression through AKT/ERK pathways. Cancer Letters. 2013; 337, 226-236.

14 Lyra-González I, Flores-Fong LE, González-García I, Medina-Preciado D, Armendáriz-Borunda J. MicroRNAs dysregulation in hepatocellular carcinoma: Insights in genomic medicine. World J Hepatol. $2015 ; 7,1530-1540$.

15 Huang JT, Liu SM, Ma H, Yang Y, Zhang X, Sun H, Zhang X, Xu J, Wang J. Systematic Review and Meta-Analysis: Circulating miRNAs for Diagnosis of Hepatocellular Carcinoma. J Cell Physiol. 2016; 231, 328-335.

16 Tsuchiya N, Sawada Y, Endo I, Saito K, Uemura Y, Nakatsura T. Biomarkers for the early diagnosis of hepatocellular carcinoma. World J Gastroenteral. 2015; 21, 10573-10583.

17 Ni W, Zhang Y, Zhan Z, Ye F, Liang Y, Huang J, Chen K, Chen LH, Ding Y. A novel IncRNA uc.134 represses hepatocellular carcinoma progression by inhibiting CUL4Amediated ubiquitination of LATS1. J Hematol Oncol. 2017; 10, 91.

18 Xing C, Lu XX, Guo PD, Shen T, Zhang S, He XS, Gan WJ, Li XM, Wang JR, Zhao YY, et al. Ubiquitinspecific protease 4-mediated Deubiquitination and stabilization of PRL-3 is required for potentiating colorectal oncogenesis. Cancer Res. 2016; 76, 83-95.

19 Meurette O, Mehlen P. Notch Signaling in the Tumor Microenvironment. Cancer Cell. 2018; 34, 536-548.

20 Vaish V, Kim J, Shim M. Jagged-2 (JAG2) enhances tumorigenicity and chemoresistance of colorectal cancer cells. Oncotarget. 2017; 8, 53262-53275. 
21 Asnaghi L, Handa JT, Merbs SL, Harbour JW, Eberhart CG. A role for Jag2 in promoting uveal melanoma dissemination and growth. Invest Ophthalmol Vis Sci. 2013; 54, 295-306.

22 Choy L, Hagenbeek TJ, Solon M, French D, Finkle D, Shelton A, Venook R, Brauer MJ, Siebel CW. Constitutive notch3 signaling promotes the growth of basal breast cancers. Cancer Res. 2017; 77, 14391452.

23 Li L, Zhao F, Lu J, Li T, Yang H, Wu C, Liu Y. Notch-1 signaling promotes the malignant features of human breast cancer through NF-kappaB activation. PLoS One. 2014; 9, e95912.

\section{Figures}
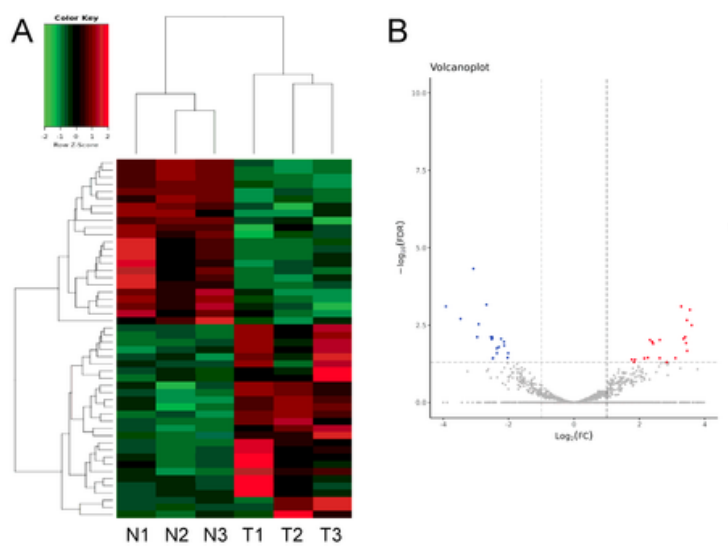

E

GO-Analysis_BP -Log10(P-value)

0.0408121 .622428323 .64
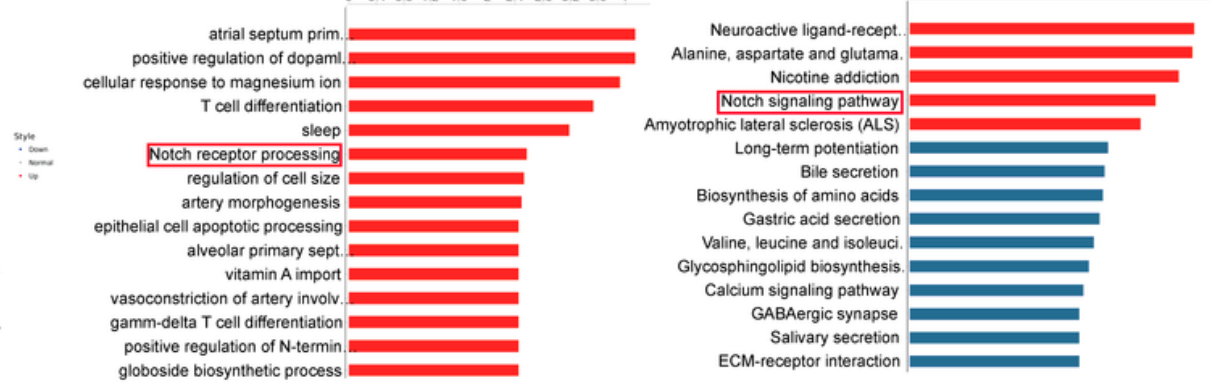

C

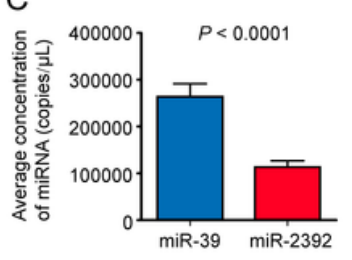

D
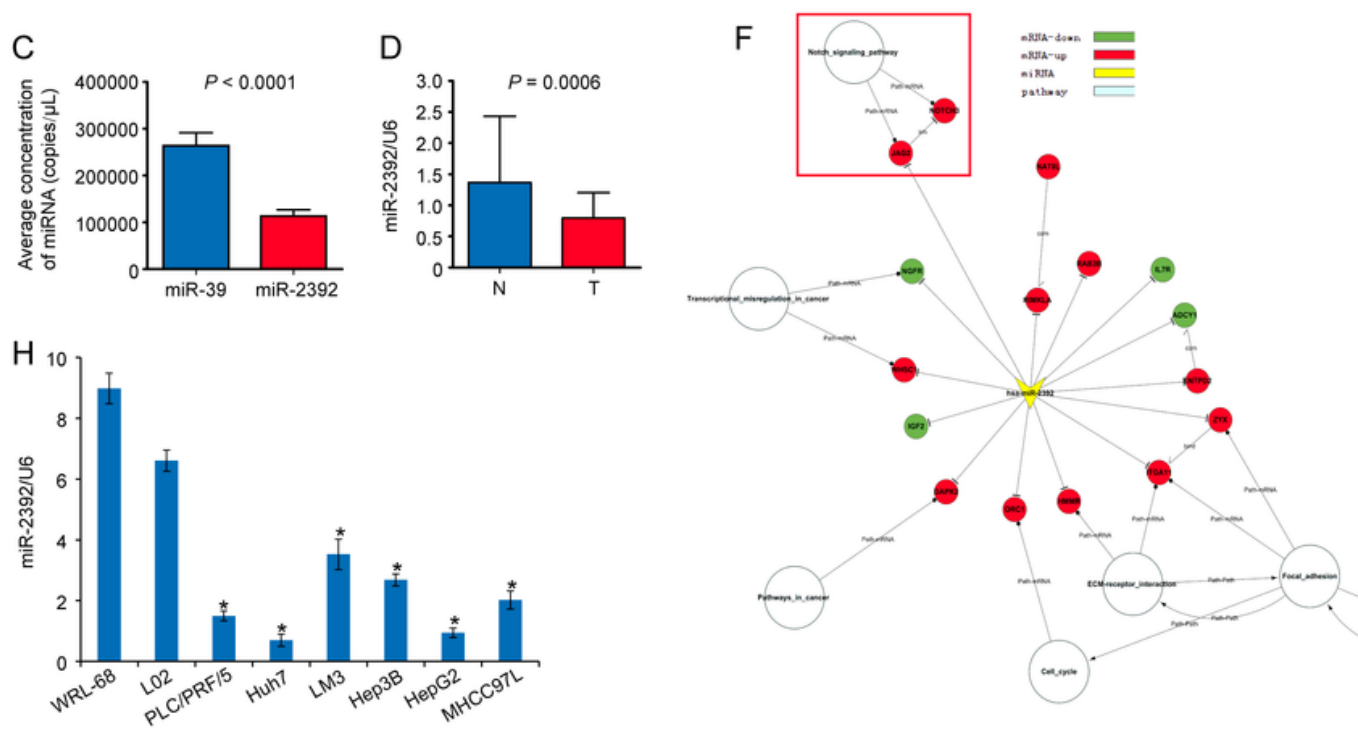

G
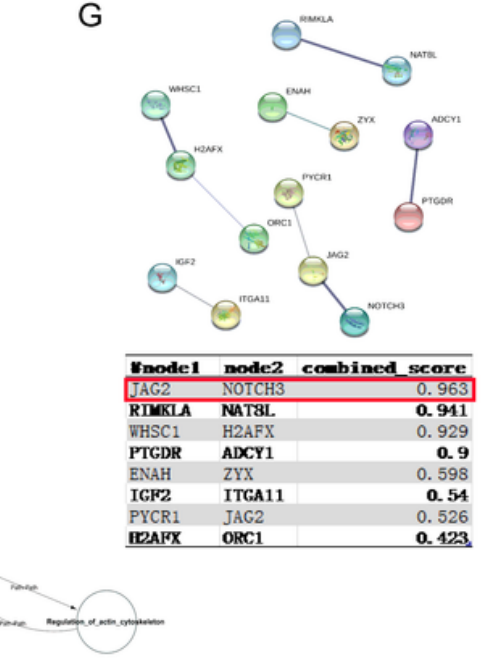

\section{Figure 1}

Differential expression and validation of miRNAs in HCC. A: Heat map representation of the dis-regulatory miRNAs involved in HCC samples (T) and paired non-tumor samples (N). Expression values are represented in shades of red and green, indicating expression above and below the median expression value across all samples B: Volcano map for alterations in miRNAs expression in HCC samples, as compared to paired non-tumor samples. Red dots denote genes with a > 2-fold change (FC) in expression. 
Blue dots denote genes with no significant change in expression $(P>0.05)$. C: The average concentration of miR-2392 in HCC patients' serum ( $n=50)$. D: The expression level of miR-2392 in HCC samples $(T)$ and paired non-tumor samples $(N)(n=50)$. E: Gene ontology $(G O)$ and pathway analysis of differentially expressed miRNAs. Top 15 significant $\mathrm{GO}$ terms (biological processes) associated with the identified differentially expressed miRNAs. The vertical axis represents the GO category and pathway category, and the horizontal axis represents the - Log10 (P-value). Greater -Log10 (P-value) scores correlated with increased statistical significance. F: Analysis of miRNA\&mRNA\&pathway network. G: Analysis of protein\&protein interaction (PPI) network. $\mathrm{H}$ : The expression level of miR-2392 in normal liver cells and HCC cells ( $\left.{ }^{*}<0.05\right)$.
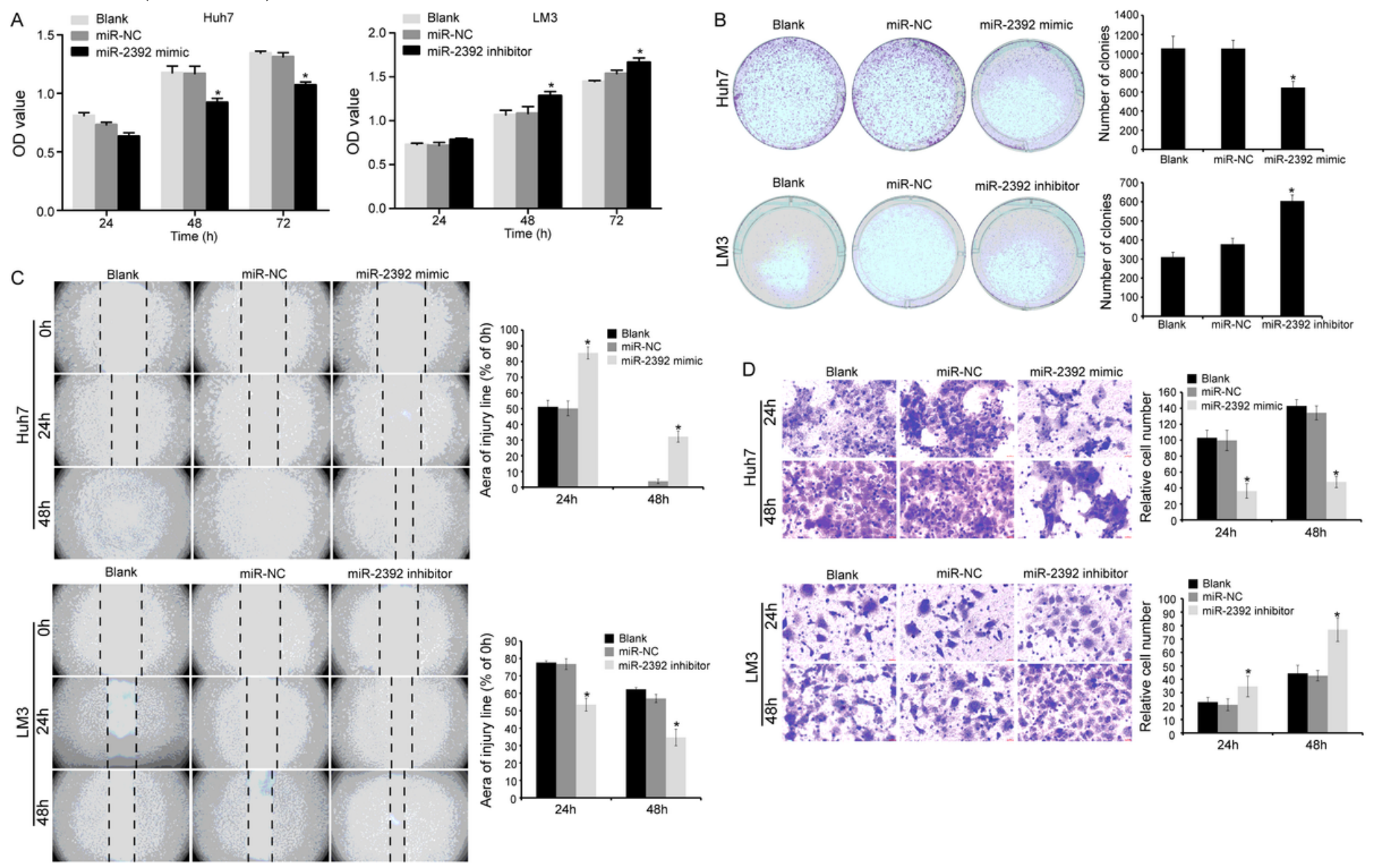

Figure 2

miR-2392 inhibits proliferation, clone formation, migration and invasion of HCC cells. A: miR-2392 significantly inhibited HCC cells growth by CCK8 assay. B: Neoplastic capacity of HCC cells was assessed by plate colony formation assay. C: Wound-healing assays were performed to assess HCC cells migration ability. Wound closure was determined at $0 \mathrm{~h}, 24 \mathrm{~h}$ and $48 \mathrm{~h}$ after the scratch. D: Representative images and quantification of transwell assays indicated the invasive capability of HCC cells. Data were presented as mean $\pm S D, * P<0.05$. 

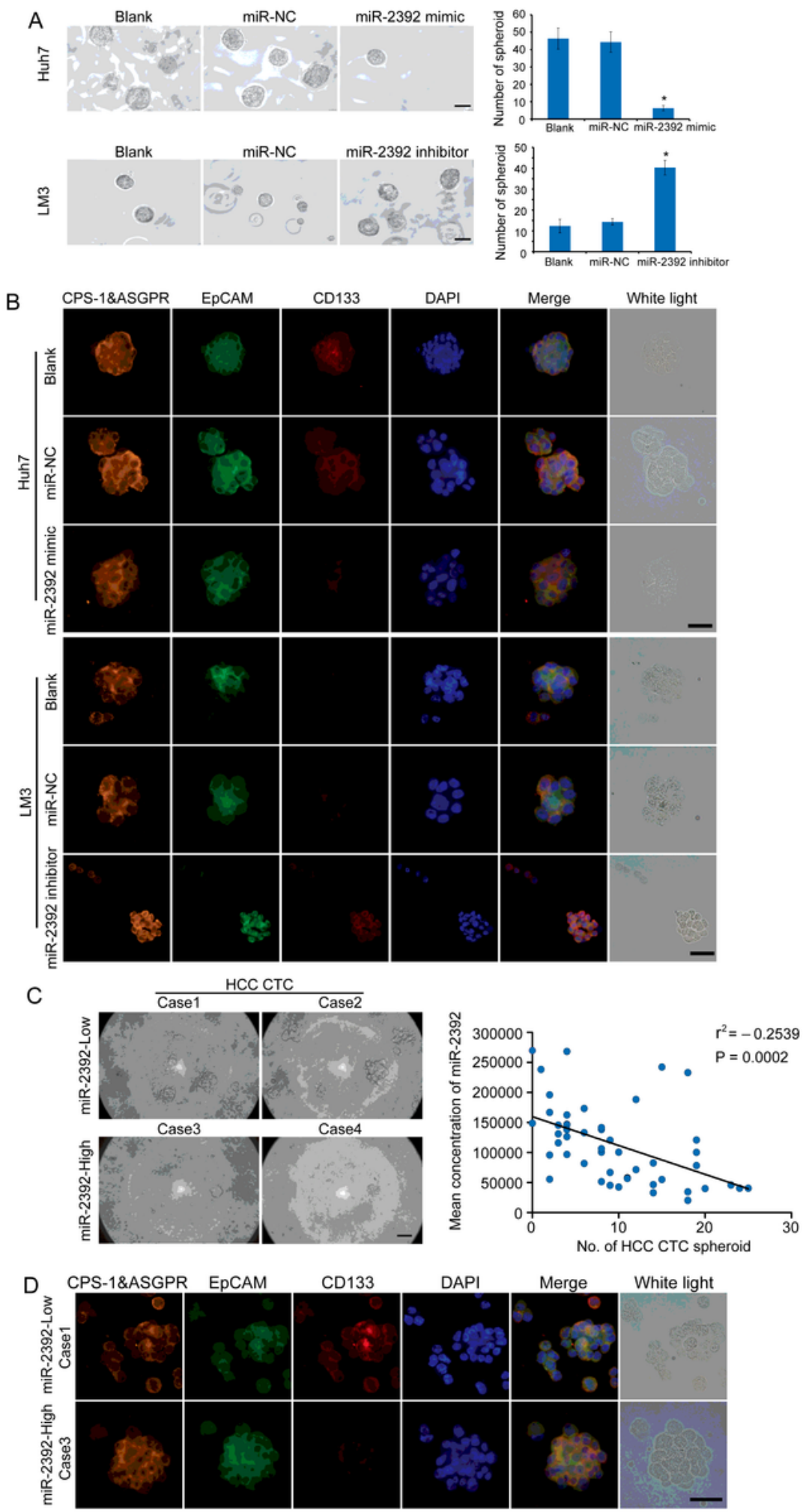

\section{Figure 3}

miR-2392 suppresses HCC cell spheroid formation and maintenance of CSC-like characteristics. A: Threedimensional spheroid assay was used to detect the spheroidizing ability of HCC cells ( $\left.{ }^{*} P<0.05\right)$, scale bar $=100 \mu \mathrm{m}$. B: Cellular immunofluorescence of hepatocyte markers (CPS1\&ASGPR) and stem cell markers (EpCAM \& CD133) in HCC cell spheroids, scale bar=100 $\mu$ m. C: Three-dimensional spheroid assay was used to detect the spheroidizing ability of HCC CTC cells, scale bar $=100 \mu \mathrm{m}$. The correlation between 
the concentration of miR-2392 in HCC patients' serum and the spheroid number of three-dimensional culture in vitro was analyzed. D: Cellular immunofluorescence of hepatocyte markers (CPS1\&ASGPR) and stem cell markers (EpCAM \& CD133) in HCC CTC spheroids, scale bar=100 $\mu \mathrm{m}$.

A

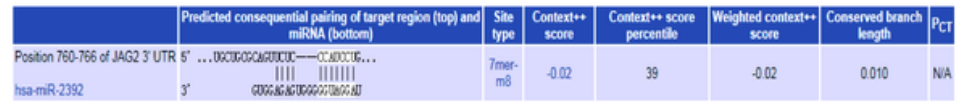

$\mathrm{B}$

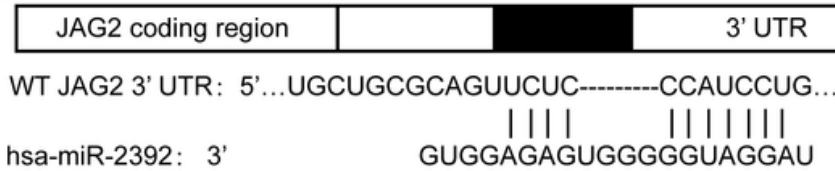

MUT JAG2 3' UTR: 5'...UGCUGCGCAGUUCUC--------AACGUUGG...

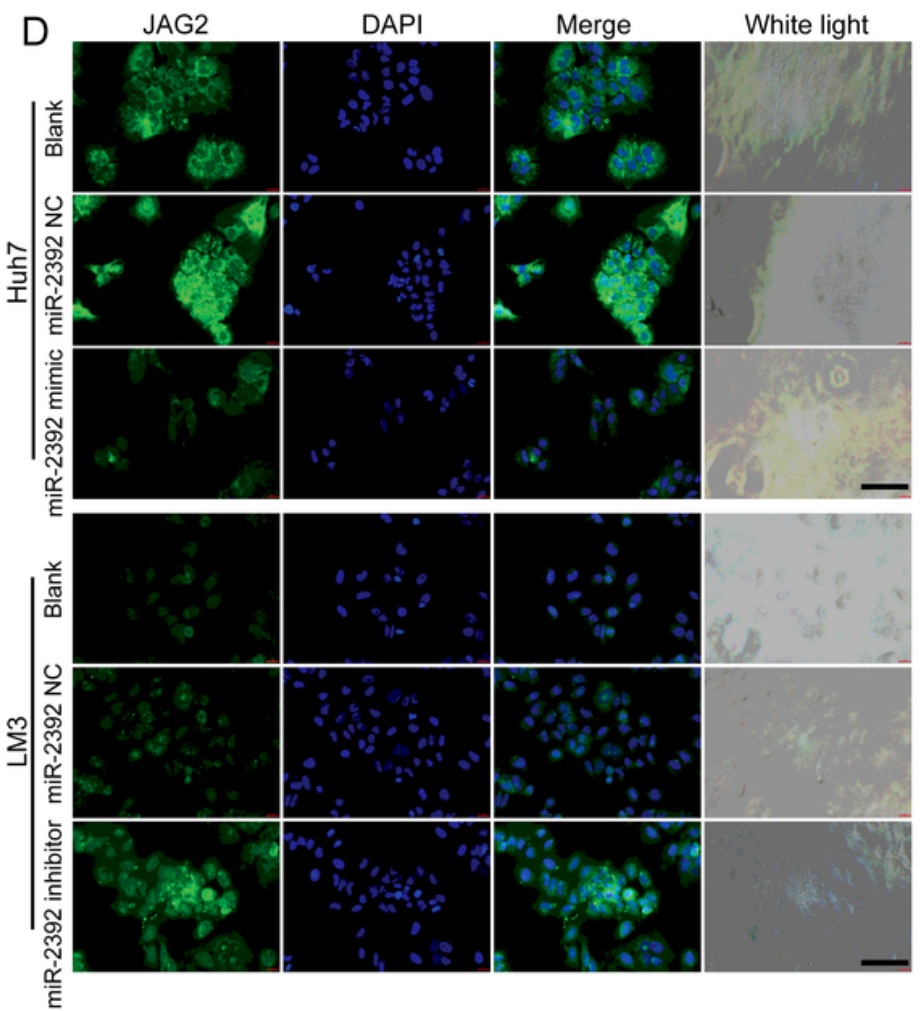

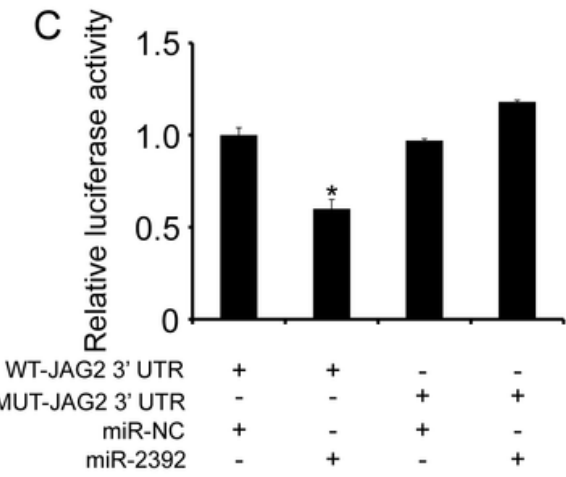
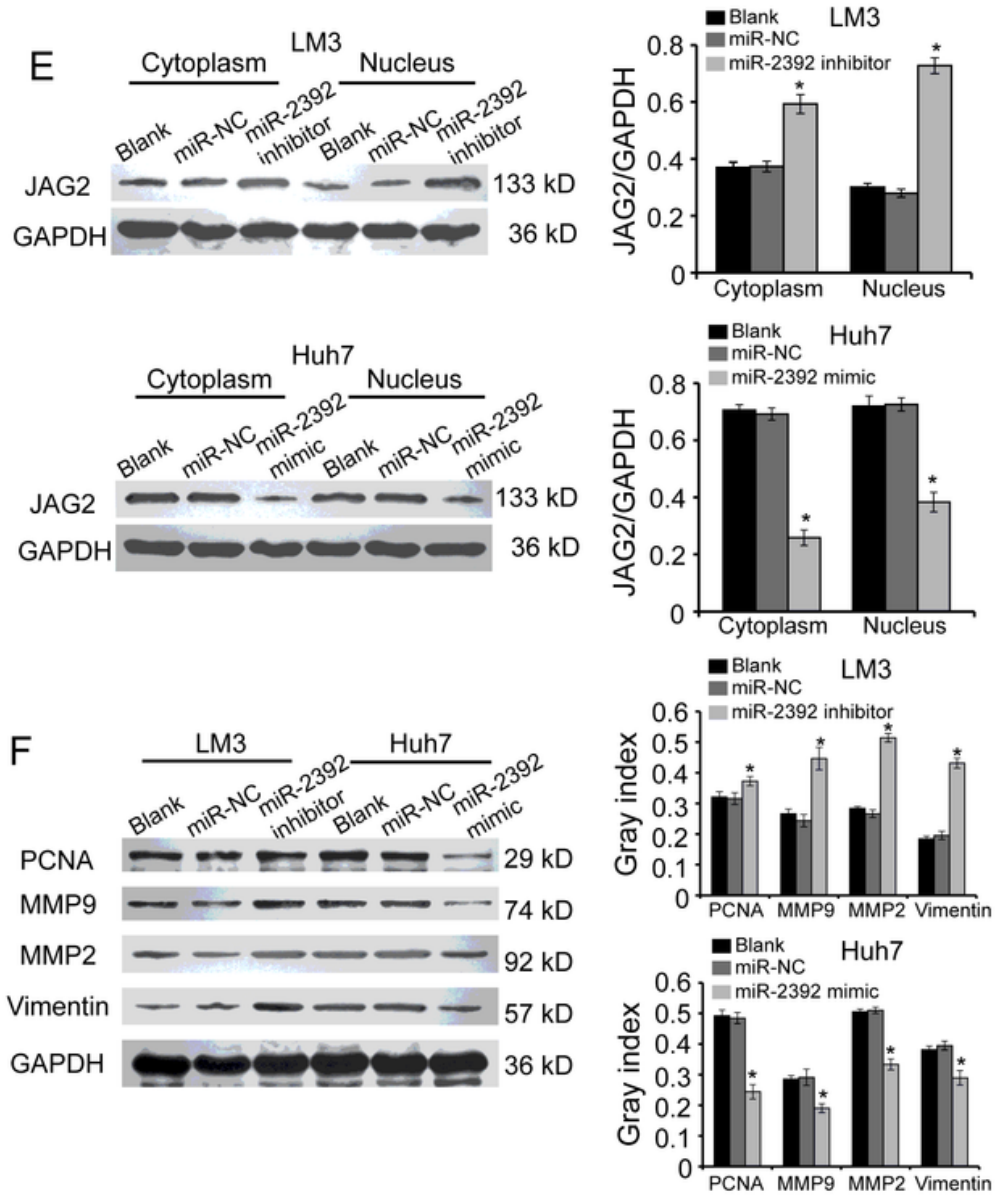

Figure 4

miR-2392 inhibit the expression and nuclear accumulation of JAG2 by targeting 3' UTR of JAG2. A: miR2392 and JAG2 have direct binding sites by prediction of Targetscan website. B: The wild-type (WT) and mutant (MUT) luciferase reporters of JAG2 were constructed. C: Luciferase reporter assay using JAG2 reporters was conducted in Huh7 cells. C: Cellular immunofluorescence was used to detect the nuclear translocation of JAG2 protein in HCC cells, scale bar=50 $\mu \mathrm{m}$. E: The protein expression of JAG2 in cytoplasm and nucleus of HCC cells was measured by western blot, $\left({ }^{*} \mathrm{P}<0.05\right)$. F: The protein expression 
of PCNA, MMP2/9 and Vimentin in cytoplasm and nucleus of HCC cells was detected by western blot, ( ${ }^{\mathrm{P}}$ $<0.05)$.
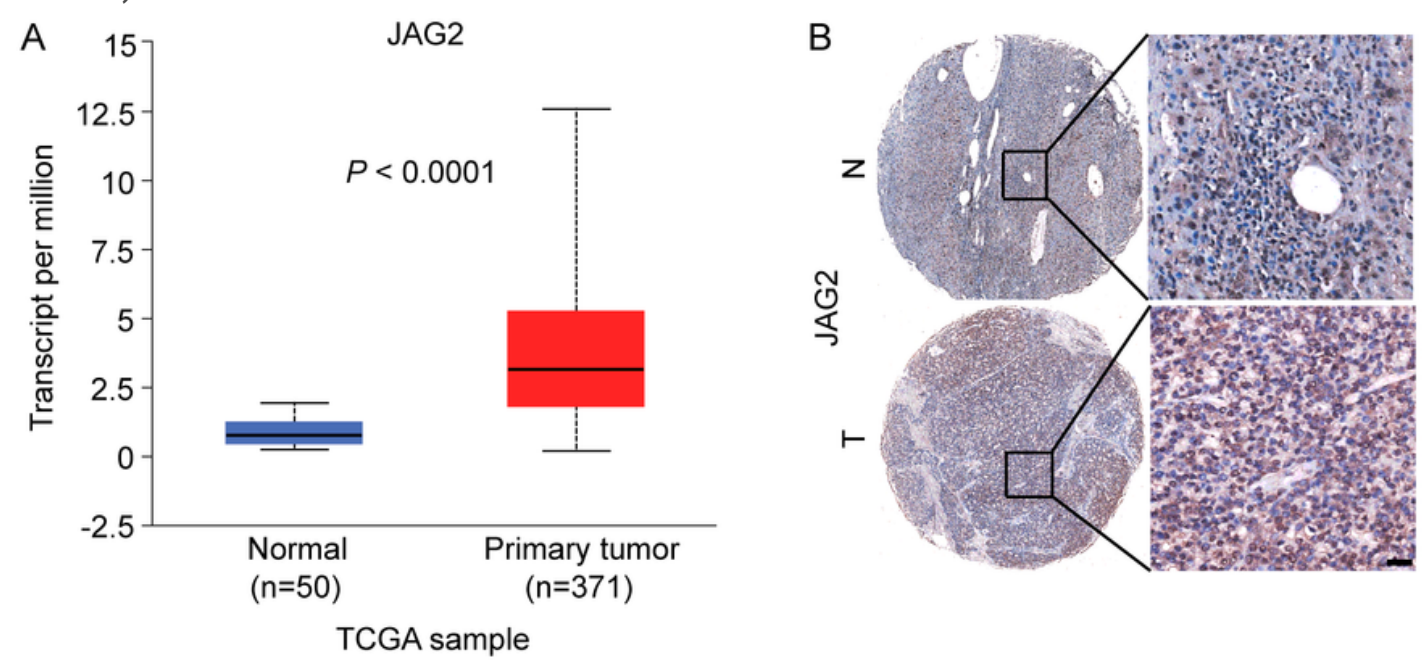

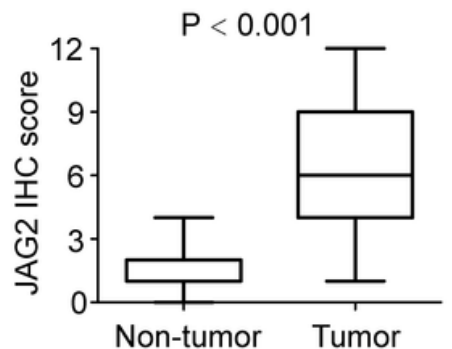

(N)

(T)

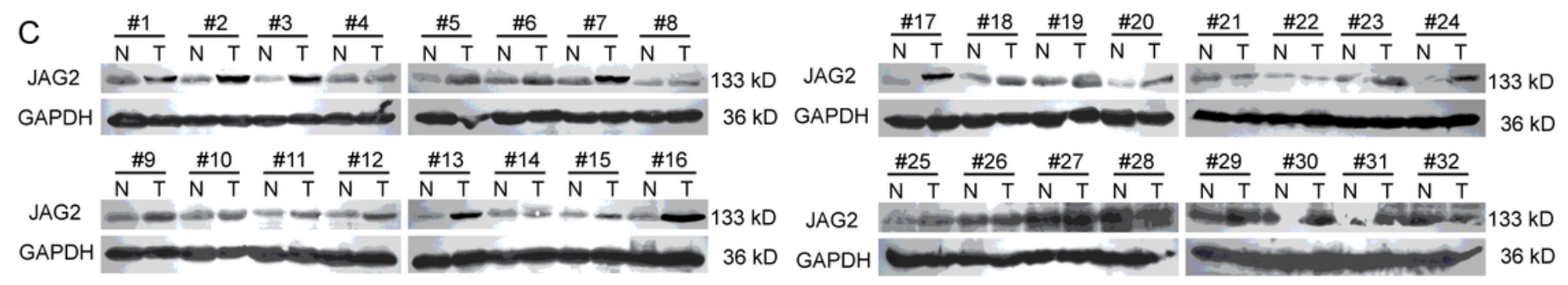

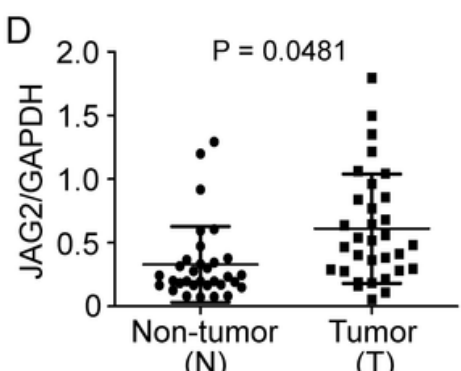

(N)
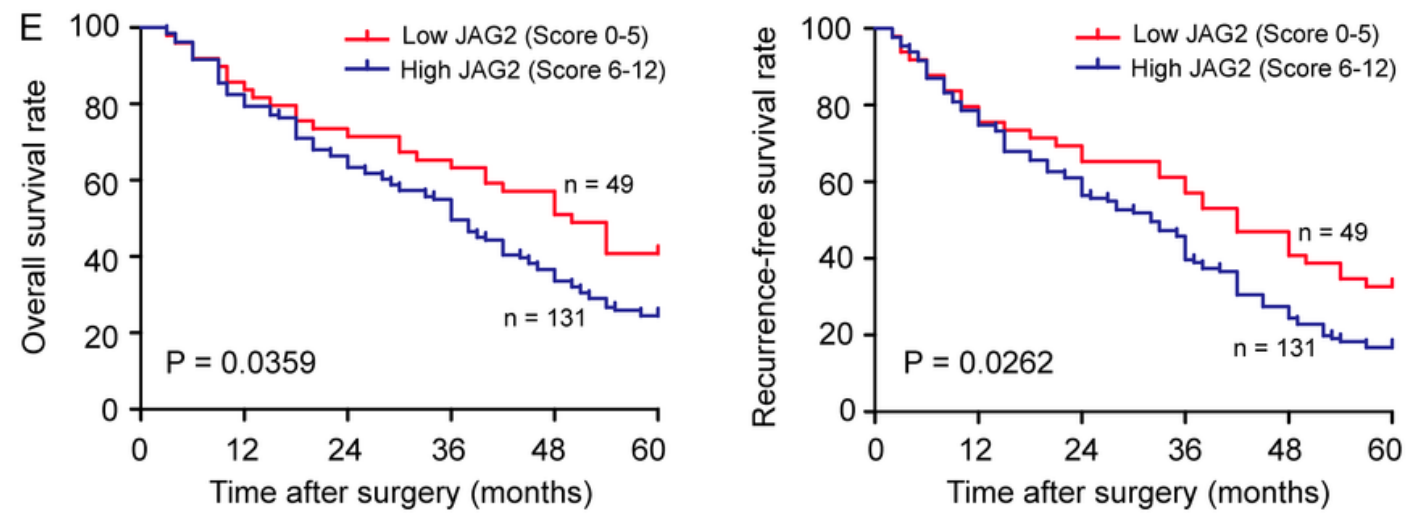

Figure 5

JAG2 is highly expressed in HCC and is closely related to poor prognosis and survival. A: JAG2 mRNA is up-regulated in HCC samples (TCGA data). B: The expression of JAG2 protein in HCC tissue microarray was detected by immunohistochemistry $(n=180)$ scale bar=50 $\mu$ m. $C$ and $D$ : Western blot of JAG2 in HCC samples $(T)$ and paired non-tumor samples $(N)(n=32)$. GAPDH was a loading control. E: The impact of JAG2 on the survival of HCC patients. 
A

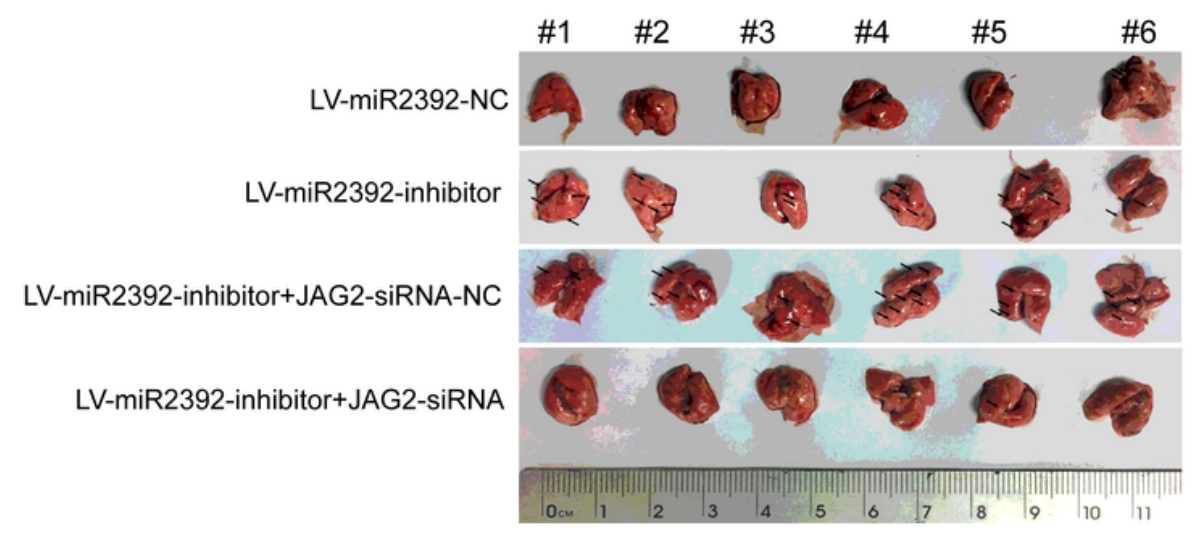

C

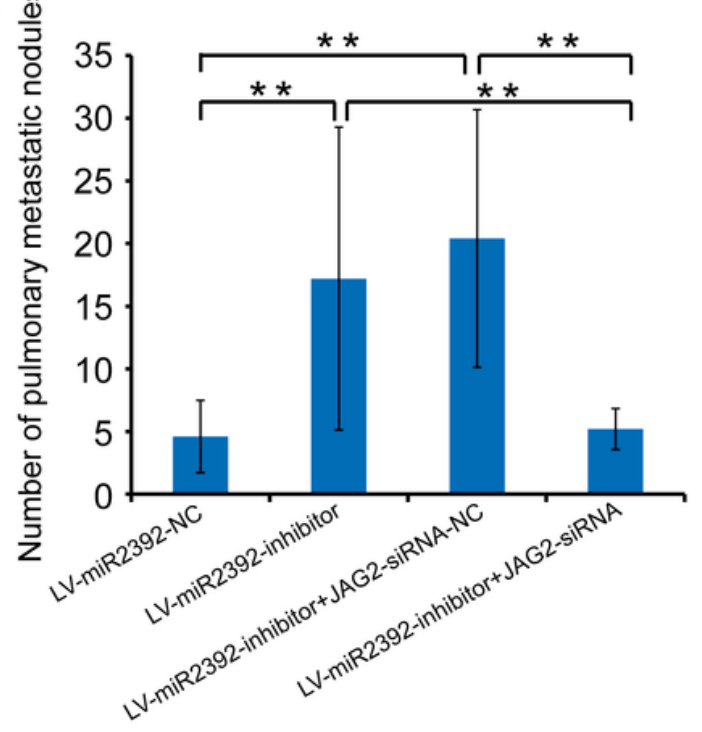

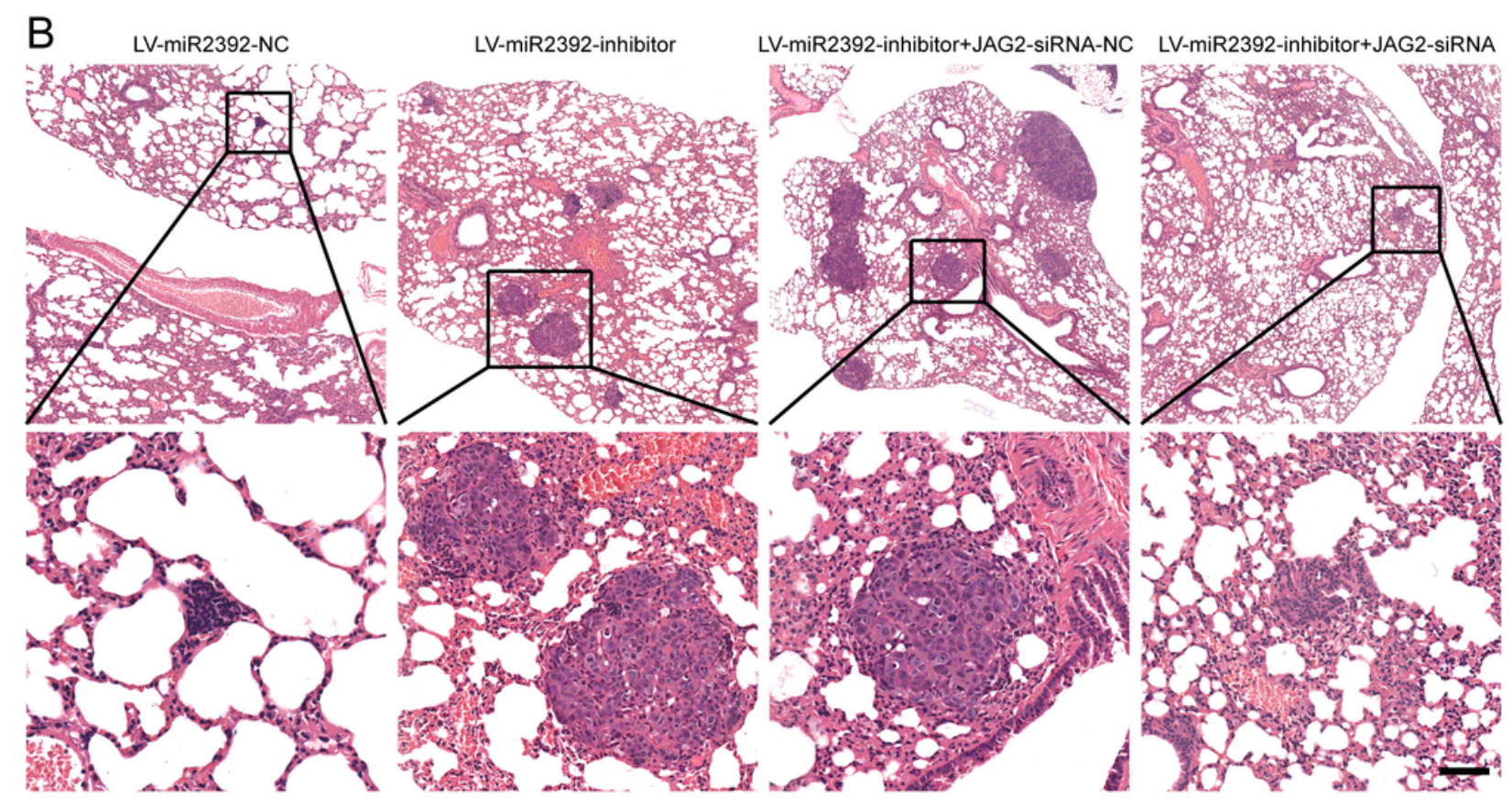

Figure 6

miR-2392 inhibits HCC cell metastasis in vivo. A: Metastatic tumor nodules in lungs of four groups of nude mice ( $n=6$ per group) (black arrow: metastatic tumor nodules). B: HE staining showed the pulmonary metastatic nodules of nude mice, scale bar $=100 \mu \mathrm{m}$. C: The numbers of pulmonary metastatic nodules in four groups $(* * \mathrm{P}<0.01)$. 


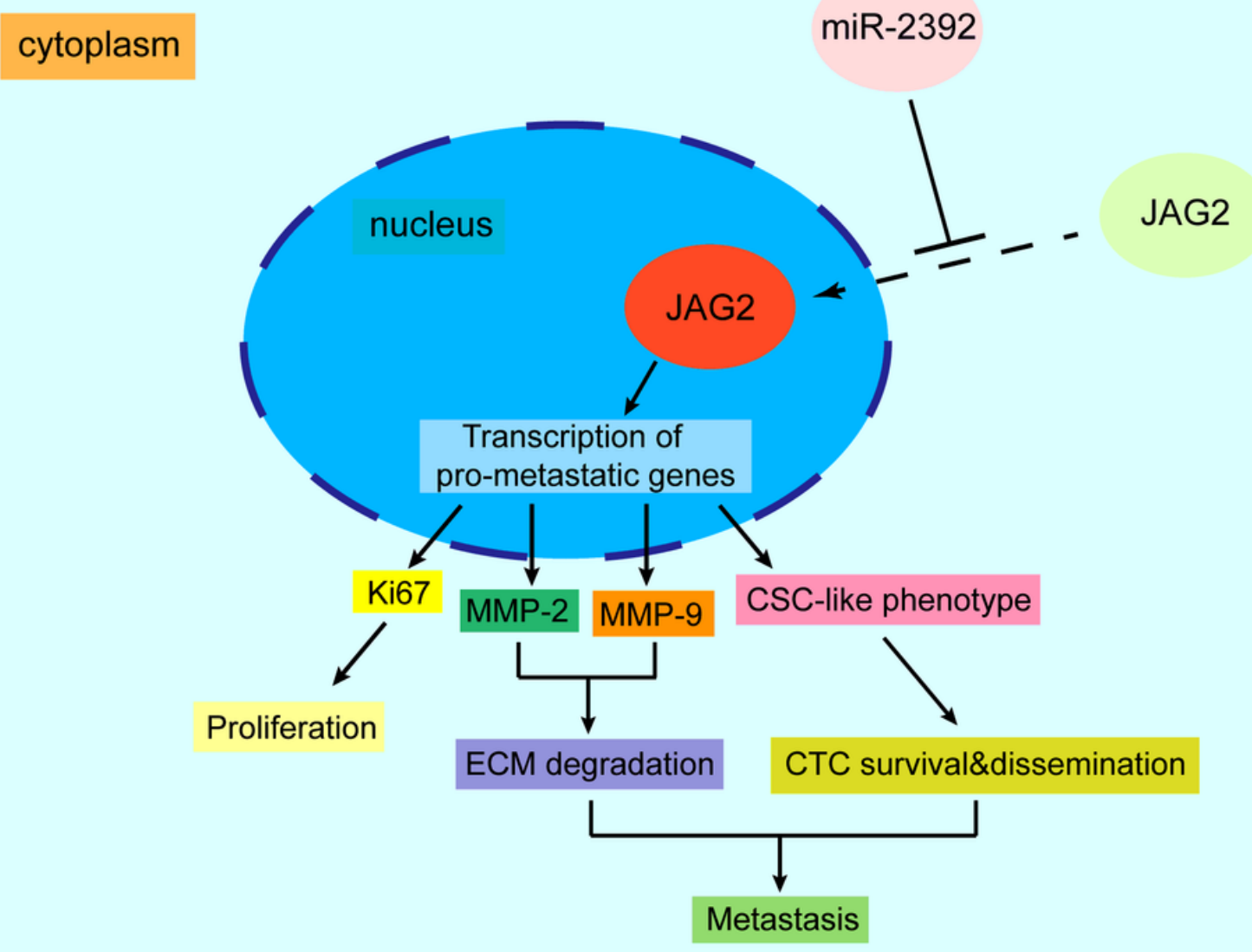

\section{Figure 7}

Schematic illustration of the mechanism of miR-2392 in HCC. Endogenous miR-2392 affects the abnormal transcription level of pro-metastatic genes in the nucleus by directly targeting JAG2 in the cytoplasm and inhibits its nuclear translocation. On the one hand, miR-2392 could rebuilt the tumor microenvironment (TME) that is prone to metastasis of HCC cells by affecting the expression levels of proliferation related gene (Ki67) and extracellular matrix degradation related indicators (MMP2 and MMP9). On the other hand, miR-2392 could transform the survival and dissemination ability of circulating tumor cells (CTCs) in the circulation system by affecting its cancer stem cells (CSC)-like characteristics and spheroidization potential, and finally limiting the metastasis of HCC.

\section{Supplementary Files}

This is a list of supplementary files associated with this preprint. Click to download.

- SupplementaryFigure1.tif

- SupplementaryFigure2.tif

- SupplementaryFigure3.tif

- SupplementaryFigure4.tif 
- SupplementaryFigure5.tif

- SupplementaryFigure6.tif

- Tables1.docx

- Tables2.docx

- TableS3.docx 